\title{
Manifestações oculares em crianças com síndrome congênita do vírus Zika
}

\section{Ocular manifestations in children with congenital Zika vírus syndrome}

Paula Yndihanara Monteiro Andrade ${ }^{1}$ Nivia Maria Rodrigues Arrais ${ }^{1}$. Cláudia Rodrigues Souza Maia ${ }^{1}$ Clicério José de Souza Rebouças ${ }^{1}$. Renackson Jordelino Garrido².

1 Universidade Federal do Rio Grande do Norte (UFRN), Natal, Rio Grande do Norte, Brasil. 2 Universidade Federal do Ceará (UFC), Fortaleza, Ceará, Brasil

\section{RESUMO}

Objetivo: Descrever o perfil epidemiológico e as alterações oculares em recém-nascidos acompanhados em um ambulatório de síndrome congênita do vírus Zika (SCZ) no Hospital Universitário Onofre Lopes (HUOL), em Natal, Rio Grande do Norte. Metodologia: Estudo retrospectivo, transversal e descritivo pela análise de prontuários de 32 pacientes com diagnóstico de SCZ submetidos a exame oftalmológico entre janeiro e agosto de 2016, no serviço de oftalmologia do HUOL. Resultados: Metade dos pacientes era do sexo masculino e a idade média ao exame foi 4,9 meses de vida. $43,7 \%$ tinham baixo peso ao nascimento e as alterações oculares mais frequentes foram atrofia macular $(36,3 \%)$ e mottling $(13,6 \%)$. As mães apresentaram mais sintomas no primeiro trimestre de gestação $(62,9 \%)$, sendo mais prevalente exantema $(92,5 \%)$ e prurido $(66,6 \%)$. Não foram encontradas evidências de diferença estatística entre a alteração ocular e as características gerais dos pacientes. Conclusões: Descrever o perfil epidemiológico e as principais alterações oftalmológicas pode guiar profissionais no diagnóstico das lesões mais prevalentes mais precocemente, possibilitando correção de lesões potencialmente curáveis e/ou ações que permitam seu crescimento e desenvolvimento com menor perda da qualidade de vida.

Palavras-chave: Zika vírus. Transmissão vertical. Manifestações oculares.

\section{ABSTRACT}

Objective: To describe the epidemiological profile and eye changes in newborns followed at a Zika virus congenital syndrome (ZCS) at Onofre Lopes University Hospital (HUOL) in Natal, Rio Grande do Norte. Methodology: Retrospective, cross-sectional and descriptive study through the analysis of medical records of 32 patients diagnosed with ZCS, who underwent eye examination between January and August 2016, at the ophthalmology service of HUOL. Results: Half of the patients were male and the mean age at examination was 4.9 months of life. $43.7 \%$ had low birth weight and the most frequent eye changes were macular atrophy (36.3\%) and mottling (13.6\%). Mothers presented more symptoms in the first trimester of pregnancy $(62.9 \%)$, being more prevalent rash $(92.5 \%)$ and pruritus $(66.6 \%)$. No evidence of statistical difference was found between ocular change and general patient characteristics. Conclusions: Describing the epidemiological profile and the main ophthalmologic alterations can guide professionals in the diagnosis of the most prevalent lesions earlier, enabling correction of potentially curable lesions and/or actions that allow their growth and development with less loss of quality of life.

Keywords: Zika virus. Vertical transmission. Eye manifestations.

Autor correspondente: Paula Yndihanara Monteiro Andrade, Rua Joaquim Inácio, 1801, Santorini Residence, Tirol, Natal, Rio Grande do Norte, Brasil. CEP: 59822-180. Telefone: +55 84 99964-7947. E-mail: paulayndihanara@gmail.com

Conflito de interesses: Não há qualquer conflito de interesses por parte de qualquer um dos autores.

Recebido em: 03 Dez 2019; Revisado em: 29 Jul 2020; Aceito em: 30 Jul 2020. 


\section{INTRODUÇÃO}

O Zika Virus (ZIKV) é um flavivirus que tem como vetor os mosquitos do gênero Aedes, isolado inicialmente na floresta Entebbe, na Uganda, em $1947 .^{1-2} \mathrm{O}$ primeiro caso de ZIKV detectado no Brasil ocorreu em março de 2015, na Bahia e a confirmação da primeira transmissão autóctone ocorreu em abril do mesmo ano. ${ }^{2}$ Foram reportados 239.742 casos até 2018, havendo um pico de disseminação nos dois primeiros anos. ${ }^{3}$

No estado do Rio Grande do Norte foram notificados 3.691 casos prováveis de febre pelo vírus Zika em 2016 e 440 em 2017 , colocando o estado em $3^{\circ}$ lugar na região na incidência por 100.000 habitantes no período. ${ }^{4} \mathrm{~A}$ infecção pode ocorrer não só através do vetor primário e transmissão vertical (TV), mas também por: via sexual, transfusão sanguínea, acidentes de laboratório. ${ }^{5}$

A doença possui amplo espectro de apresentação, com sintomas clínicos maternos: febre baixa, artralgia, rash maculopapular e cefaleia. ${ }^{5}$ Podem ocorrer ainda: mialgia, conjuntivite e linfadenopatia. ${ }^{6}$ Tais sintomas são preocupantes nas gestantes, embora a TV possa ocorrer em todos os trimestres, estando a paciente sintomática ou não, ela é especialmente importante no primeiro trimestre..$^{7-8}$

$\mathrm{O}$ vírus tem grande potencial de causar anomalias congênitas, como: alterações cerebrais estruturais, principalmente microcefalia, além de alterações oculares, contraturas congênitas e déficits neurológicos. ${ }^{8} \mathrm{O}$ tropismo oftalmológico do ZIKV, pode levar a alterações estruturais em 21,4\% a $55, \%$ dos recém-nascidos com a infecção congênita. São descritos acometimento do segmento posterior (retina, nervo óptico e vasos retinianos). Já no segmento anterior, relata-se coloboma da íris e subluxação do cristalino, podendo haver também alteração estrutural como microftalmia. Há também acometimento do nervo oculomotor (levando a nistagmo e estrabismo), além de mottling focal pigmentar, atrofia coriorretniana e macular. ${ }^{9-10}$

O objetivo do presente estudo é relatar as alterações oftalmológicas em recém-nascidos acompanhados em um ambulatório de Síndrome Congênita do Vírus Zika (SCZ) no Hospital Universitário Onofre Lopes (HUOL), em Natal, Rio Grande do Norte e comparar fatores epidemiológicos entre os lactentes que manifestaram ou não alterações no exame oftalmológico.

\section{MÉTODOS}

Estudo transversal, retrospectivo e descritivo através da análise de prontuários de 32 pacientes nascidos entre julho de 2015 e janeiro de 2016, em acompanhamento ambulatorial especializado no serviço de pediatria do HUOL devido diagnóstico de SCZ.

Foi analisada uma amostra por conveniência dos lactentes que deram entrada no serviço por meio de encaminhamento de mães com doença exantemática durante a gestação e/ou recém-nascidos portadores de microcefalia ao nascimento.
Das crianças que, após avaliação, se enquadraram no diagnóstico de SCZ foram selecionadas aquelas que fizeram exames oftalmológicos no serviço especializado. Os pacientes foram divididos em dois grupos: com e sem alterações oftalmoscópicas, para comparação.

O diagnóstico de SCZ foi realizado segundo as definições vigentes no protocolo presente no "Orientações integradas de vigilância e atenção à saúde no âmbito da emergência de saúde pública de importância nacional do Ministério da Saúde”. ${ }^{11}$

Os pacientes foram submetidos ao exame oftalmológico composto de biomicroscopia, reflexo vermelho e fundoscopia, entre janeiro e agosto de 2016, no serviço de oftalmologia do HUOL. Neste momento, foram investigados quanto a presença de catarata, alterações do nervo óptico ou da mácula e demais anormalidades.

Para esse estudo, foram colhidas nos prontuários as seguintes variáveis dos recém-nascidos: sexo, idade gestacional, estatura, peso e perímetro cefálico ao nascimento além da adequação de peso, estatura e idade gestacional. Esses três últimos foram avaliados pelos scores dos gráficos de crescimento do Multicentre Growth Reference Study (MGRS) e da OMS - 2006. ${ }^{12-13}$ Sobre as mães, buscaram-se informações dos sintomas durante a gestação como exantema, febre, artralgia, mialgia, prurido e conjuntivite e a época de aparecimento das manifestações sintomáticas.

A partir da suspeição diagnóstica, um parente direto (pai ou mãe), foi solicitado a comparecer no ambulatório para assinar um termo de consentimento livre e esclarecido, de número 57444016.1.0000.5292 do Comitê de Ética da comissão do HUOL. A pesquisa esteve de acordo com a resolução 466/12 do Conselho Nacional de Saúde e com os critérios estabelecidos pela Declaração de Helsinki.

Os dados foram tabulados no software Excel 2010, para Windows ${ }^{\circledR}$ e as análises das tabelas descritivas e aplicação de testes estatísticos por meio do software Statistical Package for the Social Sciences, SPSS, versão 20.0.0.

Foram excluídos da base de dados os lactentes que apresentaram confirmação de outras causas para a microcefalia e/ou títulos persistentemente elevados de anti-IgG ou presença de anticorpos IgM específicos para toxoplasmose, citomegalovírus, vírus herpes simples, HIV ou rubéola e/ou história familiar de microcefalia.

\section{RESULTADOS}

O estudo compreendeu a investigação de 32 lactentes, os quais, $11(34,4 \%)$ apresentaram alterações oculares. Os exames oftalmológicos foram realizados entre 1,2 e 9 meses de vida (média de 4,9 meses).

De acordo com o protocolo do Ministério da Saúde para o diagnóstico de síndrome congênita, ${ }^{11}$ duas $(6,2 \%)$ crianças 
apresentaram infecção congênita com identificação sorológica, trinta $(93,7 \%)$ possuíam infecção congênita sem identificação etiológica, sendo estas, vinte e seis $(86,6 \%)$ casos confirmados e quatro $(13,3 \%)$ casos prováveis de infecção congênita sem identificação etiológica.

Metade dos pacientes eram do sexo masculino. Quanto ao peso ao nascimento, $43,7 \%$ tiveram peso menor de 2.500 gramas (baixo peso ao nascer). A adequação do peso para a idade gestacional, teve a seguinte distribuição: $>-2(62,5 \%)$, entre -3 e $-2(21,8 \%)$ e $<-3(15,6 \%)$. Com relação ao escore perímetro cefálico, $50,0 \%$ foram $<-3,34,3 \%$ entre -3 e -2 e
$15,6 \%>-2$ (normal). Em relação ao escore da estatura: $>-2$ $(43,7 \%)$, entre - 3 e $-2(21,8 \%)$ e $<-3(34,3 \%)$. Quanto a idade gestacional, a maioria dos recém-nascidos eram termos $(84,3)$ e $15,6 \%$ pré-termos (Tabela 01 ).

A maioria das mães $(84,4 \%)$ referiram sintomas de infecção durante a gestação, sendo $62,9 \%$ no primeiro trimestre e $29,6 \%$ no $2^{\circ}$ trimestre, sendo os mais prevalentes exantema $(92,6 \%)$, prurido $(66,7 \%)$ e febre $(51,9 \%)$. Também foram relatados, em menor escala artralgia, mialgia e conjuntivite (Tabela 01).

Tabela 1. Perfil geral dos pacientes.

\begin{tabular}{|c|c|c|c|}
\hline Características & & Frequência & $\%$ \\
\hline \multirow{2}{*}{ Sexo } & Masculino & 16 & 50,00 \\
\hline & Feminino & 16 & 50,00 \\
\hline \multirow{2}{*}{ Grupo } & Sem alteração & 21 & 65,62 \\
\hline & Com alteração & 11 & 34,38 \\
\hline \multirow{2}{*}{ Peso ao nascimento } & $<2.500$ & 14 & 43,75 \\
\hline & $>2.500$ & 18 & 56,25 \\
\hline \multirow{3}{*}{ Escore peso } & $>-2$ & 20 & 62,50 \\
\hline & Entre -3 e -2 & 7 & 21,87 \\
\hline & $<-3$ & 5 & 15,63 \\
\hline \multirow{3}{*}{ Escore perímetro cefálico } & $>-2$ & 5 & 15,63 \\
\hline & Entre -3 e -2 & 11 & 34,38 \\
\hline & $<-3$ & 16 & 50,00 \\
\hline \multirow{3}{*}{ Escore estatura } & $>-2$ & 14 & 43,74 \\
\hline & Entre -3 e -2 & 7 & 21,88 \\
\hline & $<-3$ & 11 & 34,38 \\
\hline \multirow{2}{*}{ Idade gestacional } & Pré termo & 5 & 15,63 \\
\hline & Termo & 27 & 84,37 \\
\hline \multirow{2}{*}{ Sintomas maternos } & Sim & 27 & 84,37 \\
\hline & Não & 5 & 15,63 \\
\hline \multirow{3}{*}{$\begin{array}{l}\text { Trimestre de sintomas } \\
\text { maternos }\end{array}$} & $1^{\mathrm{o}}$ & 17 & 62,96 \\
\hline & $2^{o}$ & 8 & 29,63 \\
\hline & $3^{\circ}$ & 2 & 7,41 \\
\hline \multirow{6}{*}{$\begin{array}{l}\text { Principais sintomas maternos } \\
\text { (Múltipla Resposta) }\end{array}$} & Exantema & 25 & 92,59 \\
\hline & Prurido & 18 & 66,67 \\
\hline & Febre & 14 & 51,85 \\
\hline & Artralgia & 11 & 40,74 \\
\hline & Mialgia & 9 & 33,33 \\
\hline & Conjuntivite & 1 & 3,70 \\
\hline
\end{tabular}


Para verificar a normalidade dos dados aplicou-se o teste de Shapiro Wilks, atribuindo um nível de significância de 5\%. Observou-se evidência estatística que o escore do perímetro cefálico e idade gestacional não possuem distribuição normal, sendo recomendável a aplicação de testes estatísticos não paramétricos.

Através do teste estatístico não paramétrico de Mann-Whitney, para um nível de significância de 5\%, houve evidência de diferença estatística entre o grupo em estudo com relação à idade gestacional, onde os pacientes com alteração ocular apresentaram maior idade gestacional $(p<0,05)$ (Tabela 02). Aplicando teste qui-quadrado $\left(\boldsymbol{X}^{2}\right)$ ou exato de Fisher, não foram constatadas evidências de diferença estatística entre a alteração ocular e as características gerais dos pacientes (Tabela 03).

Dos 11 pacientes estudados, ou seja, 22 olhos, o nervo óptico apresentou-se pálido em $2(9,0 \%)$, e pequeno e pouco corado em $1(4,5 \%)$. Foi encontrada atrofia macular isolada em 8 olhos (36,3\%), mottling em $3(13,6 \%)$ e coloboma em mácula temporal, atrofia pigmentar, alteração de pigmentação e atrofia geográfica em 1 olho cada (4,5\%). Da associação de alterações, atrofia macular e mottling foi descrita em 2 olhos (9\%), e coloboma atrelado a alteração pigmentar, atrofia retiniana concomitante a atrofia pigmentar foram relatadas em 1 olho $(4,5 \%)$ cada. Nenhum dos pacientes apresentou catarata (Tabela 04).

Tabela 2. Comparação das variáveis clínicas do paciente com relação ao grupo de com e sem alteração.

\begin{tabular}{|c|c|c|c|c|c|c|c|c|c|c|}
\hline Variáveis & Grupo & Mínimo & Máximo & $25 \%$ & Mediana & $75 \%$ & Média & DP & $\mathrm{CV}$ & Valor - p \\
\hline \multirow{2}{*}{$\begin{array}{l}\text { Peso } \\
\text { nascimento }\end{array}$} & C.A & 1920,00 & 3645,00 & 2116,00 & 2754,00 & 3300,00 & 2702,73 & 631,09 & 23,35 & \multirow{2}{*}{$0,846^{(1)}$} \\
\hline & S.A & 1590,00 & 4140,00 & 2300,00 & 2750,00 & 3216,00 & 2747,95 & 616,07 & 22,42 & \\
\hline \multirow{2}{*}{$\begin{array}{l}\text { Escore peso } \\
\text { ao nascer }\end{array}$} & C.A & $-3,00$ & 1,00 & $-3,00$ & $-1,00$ & 0,00 & $-1,36$ & 1,50 & $-110,11$ & \multirow{2}{*}{$0,819^{(1)}$} \\
\hline & S.A & $-4,00$ & 2,00 & $-2,00$ & $-1,00$ & 0,00 & $-1,24$ & 1,45 & $-116,78$ & \\
\hline \multirow{2}{*}{$\begin{array}{l}\text { Escore } \\
\text { perímetro } \\
\text { cefálico }\end{array}$} & C.A & $-7,00$ & $-1,00$ & $-4,00$ & $-4,00$ & $-2,00$ & $-3,45$ & 1,86 & $-53,94$ & \multirow{2}{*}{$0,254^{(2)}$} \\
\hline & S.A & $-9,00$ & $-2,00$ & $-6,00$ & $-4,00$ & $-3,00$ & $-4,33$ & 1,96 & $-45,18$ & \\
\hline \multirow{2}{*}{$\begin{array}{l}\text { Escore } \\
\text { estatura }\end{array}$} & C.A & $-8,00$ & 0,00 & $-5,00$ & $-3,00$ & $-1,00$ & $-3,09$ & 2,63 & $-84,93$ & \multirow{2}{*}{$0,313^{(1)}$} \\
\hline & S.A & $-5,00$ & 1,00 & $-3,00$ & $-2,00$ & $-1,00$ & $-2,19$ & 1,50 & $-68,66$ & \\
\hline \multirow{2}{*}{$\begin{array}{l}\text { Idade } \\
\text { gestacional }\end{array}$} & C.A & 37,00 & 41,00 & 39,00 & 40,00 & 40,00 & 39,55 & 1,13 & 2,85 & \multirow{2}{*}{$0,025^{(2)}$} \\
\hline & S.A & 33,00 & 41,00 & 37,00 & 38,00 & 40,00 & 38,10 & 1,92 & 5,04 & \\
\hline
\end{tabular}

Legenda: DP: Desvio Padrão CV: Coeficiente de Variação (1) Teste de t de Student (2) Mann-Whitney.

Tabela 3. Grupo em estudo versus característica do paciente.

\begin{tabular}{|c|c|c|c|c|c|}
\hline \multirow{2}{*}{\multicolumn{2}{|c|}{ Características gerais }} & \multicolumn{2}{|c|}{ Grupo } & \multirow{2}{*}{ Total } & \multirow{2}{*}{ Valor-p } \\
\hline & & Com alteração & Sem alteração & & \\
\hline \multirow[t]{2}{*}{ Sexo } & Feminino & $43,75 \%(n=7)$ & $56,25 \%(\mathrm{n}=9)$ & $100,00 \%(n=16)$ & \multirow{2}{*}{$0,264^{(1)}$} \\
\hline & Masculino & $25,00 \%(n=4)$ & $75,00 \%(n=12)$ & $100,00 \%(n=16)$ & \\
\hline \multirow[t]{2}{*}{ Peso } & $<2.500$ & $35,71 \%(\mathrm{n}=5)$ & $64,29 \%(\mathrm{n}=9)$ & $100,00 \%(n=14)$ & \multirow{2}{*}{$1,000^{(2)}$} \\
\hline & $>2.500$ & $33,33 \%(n=6)$ & $66,67 \%(n=12)$ & $100,00 \%(n=18)$ & \\
\hline \multirow[t]{3}{*}{ Escore peso } & $>-2$ & $30,00 \%(n=6)$ & $70,00 \%(n=14)$ & $100,00 \%(n=20)$ & \multirow{3}{*}{$0,7933^{(1)}$} \\
\hline & Entre -3 e -2 & $42,86 \%(n=3)$ & $57,14 \%(\mathrm{n}=4)$ & $100,00 \%(n=7)$ & \\
\hline & $<-3$ & $40,00 \%(n=2)$ & $60,00 \%(n=3)$ & $100,00 \%(n=5)$ & \\
\hline \multirow{3}{*}{$\begin{array}{l}\text { Escore perímetro } \\
\text { cefálico }\end{array}$} & $>-2$ & $60,00 \%(n=3)$ & $40,00 \%(\mathrm{n}=2)$ & $100,00 \%(n=5)$ & \multirow{3}{*}{$0,413^{(1)}$} \\
\hline & Entre -3 e -2 & $27,27 \%(n=3)$ & $72,73 \%(n=8)$ & $100,00 \%(n=11)$ & \\
\hline & $<-3$ & $31,25 \%(n=5)$ & $68,75 \%(n=11)$ & $100,00 \%(n=16)$ & \\
\hline
\end{tabular}

Continua. 
Conclusão.

Tabela 3. Grupo em estudo versus característica do paciente.

\begin{tabular}{|c|c|c|c|c|c|}
\hline \multirow{2}{*}{ Características gerais } & & \multicolumn{2}{|c|}{ Grupo } & \multirow{2}{*}{ Total } & \multirow{2}{*}{ Valor-p } \\
\hline & & Com alteração & Sem alteração & & \\
\hline \multirow[t]{3}{*}{ Escore estatura } & $>-2$ & $35,71 \%(n=5)$ & $64,29 \%(n=9)$ & $100,00 \%(n=14)$ & \multirow{3}{*}{$0,059^{(1)}$} \\
\hline & Entre -3 e -2 & $0,00 \%(n=0)$ & $100,00 \%(n=7)$ & $100,00 \%(n=7)$ & \\
\hline & $<-3$ & $54,55 \%(n=6)$ & $45,45 \%(n=5)$ & $100,00 \%(n=11)$ & \\
\hline \multirow[t]{2}{*}{ Idade gestacional } & Pré termo & $20,00 \%(n=1)$ & $80,00 \%(n=4)$ & $100,00 \%(n=5)$ & \multirow{2}{*}{$0,637^{(1)}$} \\
\hline & Termo & $37,04 \%(n=10)$ & $62,96 \%(n=17)$ & $100,00 \%(n=27)$ & \\
\hline \multirow[t]{2}{*}{ Sintomas maternos } & Sim & $33,33 \%(n=9)$ & $66,67 \%(n=18)$ & $100,00 \%(n=27)$ & \multirow{2}{*}{$1,000^{(2)}$} \\
\hline & Não & $40,00 \%(n=2)$ & $60,00 \%(n=3)$ & $100,00 \%(n=5)$ & \\
\hline \multirow{3}{*}{$\begin{array}{l}\text { Sintomas maternos } \\
\text { (Trimestre) }\end{array}$} & $\mathbf{1}^{\mathbf{o}}$ & $41,18 \%(n=7)$ & $58,82 \%(n=10)$ & $100,00 \%(n=17)$ & \multirow{3}{*}{$0,319^{(1)}$} \\
\hline & $2^{\circ}$ & $12,50 \%(n=1)$ & $87,50 \%(n=7)$ & $100,00 \%(n=8)$ & \\
\hline & $3^{\circ}$ & $50,00 \%(n=1)$ & $50,00 \%(n=1)$ & $100,00 \%(n=2)$ & \\
\hline \multirow[t]{2}{*}{ Exantema } & Sim & $32,00 \%(n=8)$ & $68,00 \%(n=17)$ & $100,00 \%(n=25)$ & \multirow{2}{*}{$1,000^{(2)}$} \\
\hline & Não & $50,00 \%(n=1)$ & $50,00 \%(n=1)$ & $100,00 \%(n=2)$ & \\
\hline \multirow[t]{2}{*}{ Febre } & Sim & $35,71 \%(n=5)$ & $64,29 \%(n=9)$ & $100,00 \%(n=14)$ & \multirow{2}{*}{$1,000^{(2)}$} \\
\hline & Não & $30,77 \%(n=4)$ & $69,23 \%(n=9)$ & $100,00 \%(n=13)$ & \\
\hline \multirow[t]{2}{*}{ Mialgia } & Sim & $11,11 \%(n=1)$ & $88,89 \%(n=8)$ & $100,00 \%(n=9)$ & \multirow{2}{*}{$0,193^{(2)}$} \\
\hline & Não & $44,44 \%(n=8)$ & $55,56 \%(n=10)$ & $100,00 \%(n=18)$ & \\
\hline \multirow[t]{2}{*}{ Conjuntivite } & Sim & $100,00 \%(n=1)$ & $0,00 \%(n=27)$ & $100,00 \%(n=1)$ & \multirow{2}{*}{$0,333^{(2)}$} \\
\hline & Não & $30,77 \%(n=8)$ & $69,23 \%(n=18)$ & $100,00 \%(n=26)$ & \\
\hline \multirow[t]{2}{*}{ Artralgia } & Sim & $45,45 \%(n=5)$ & $54,55 \%(n=6)$ & $100,00 \%(n=11)$ & \multirow{2}{*}{$0,411^{(2)}$} \\
\hline & Não & $25,00 \%(n=4)$ & $75,00 \%(n=12)$ & $100,00 \%(n=16)$ & \\
\hline \multirow[t]{2}{*}{ Prurido } & Sim & $33,33 \%(n=6)$ & $66,67 \%(n=12)$ & $100,00 \%(n=18)$ & \multirow{2}{*}{$1,000^{(2)}$} \\
\hline & Não & $33,33 \%(n=3)$ & $66,67 \%(n=6)$ & $100,00 \%(n=9)$ & \\
\hline
\end{tabular}

Tabela 4. Achados da fundoscopia em recém-nascidos com síndrome congênita do Zika vírus.

\begin{tabular}{|c|c|c|c|c|c|c|c|c|c|c|c|c|c|c|c|c|c|c|c|c|c|c|}
\hline $\mathrm{RN}$ & 1 & 1 & 2 & 2 & 3 & 3 & 4 & 4 & 5 & 5 & 6 & 6 & 7 & 7 & 8 & 8 & 9 & 9 & 10 & 10 & 11 & 11 \\
\hline Olho & $\mathrm{D}$ & $\mathrm{E}$ & $\mathrm{D}$ & $\mathrm{E}$ & $\mathrm{D}$ & $\mathrm{E}$ & $\mathrm{D}$ & $\mathrm{E}$ & $\mathrm{D}$ & $\mathrm{E}$ & $\mathrm{D}$ & $\mathrm{E}$ & $\mathrm{D}$ & $\mathrm{E}$ & $\mathrm{D}$ & $\mathrm{E}$ & $\mathrm{D}$ & $\mathrm{E}$ & $\mathrm{D}$ & $\mathrm{E}$ & $\mathrm{D}$ & $\mathrm{E}$ \\
\hline \multicolumn{23}{|l|}{ Catarata } \\
\hline Palidez do nervo óptico & & & & & & & & & $\mathrm{x}$ & $\mathrm{x}$ & & & & & & & & & & & & \\
\hline Atrofia peripapilar & & & & & & & & & & & $\mathrm{x}$ & $\mathrm{x}$ & & & & & & & $\mathrm{x}$ & $\mathrm{x}$ & & \\
\hline Atrofia macular & & $\mathrm{x}$ & & & $\mathrm{x}$ & $\mathrm{x}$ & & $\mathrm{x}$ & $\mathrm{x}$ & $\mathrm{x}$ & & & $\mathrm{x}$ & $\mathrm{x}$ & & & & & & & & \\
\hline Atrofia geográfica & & & & & & & & & & & $\mathrm{x}$ & & & & & & & & & & & \\
\hline Atrofia retiniana & & & & & & & & & & & & & & & & & & & & & $\mathrm{x}$ & \\
\hline Atrofia pigmentar & & & & & & & & & & & & & & & & & & & & & $\mathrm{x}$ & $\mathrm{x}$ \\
\hline Coloboma & & & & $\mathrm{x}$ & & & & & & & & & & & & & $\mathrm{x}$ & & & & & \\
\hline Alteração de pigmentação & & & & & & & $\mathrm{x}$ & & & & & & & & & & $\mathrm{x}$ & & & & & \\
\hline Mottling & & & & & & & & & $\mathrm{x}$ & $\mathrm{x}$ & & $\mathrm{x}$ & & & $\mathrm{x}$ & & & $\mathrm{x}$ & & & & \\
\hline
\end{tabular}




\section{DISCUSSÃO}

A epidemia brasileira de ZIKV foi a primeira a detectar o RNA viral em amostras de líquido amniótico e no tecido cerebral de recém-nascidos e natimortos com microcefalia de mães infectadas pelo vírus durante a gestação. ${ }^{14}$ Tal detecção, permitiu dar início às investigações para estabelecer uma associação entre a infecção intrauterina e alterações cerebrais estruturais como microcefalia e também lesões oculares. ${ }^{15}$

Posteriormente, foram bem estabelecidas as desordens possíveis na SCZ: calcificações cerebrais, ventriculomegalia, alterações articulares, anormalidades oculares e microcefalia. ${ }^{16}$

Como o Rio Grande do Norte foi o segundo colocado entre os estados brasileiros com os maiores índices de infecções por ZIKV, ${ }^{4}$ é importante investigar, analisar e descrever as alterações oculares congênitas em recém-nascidos portadores de SCZ. 34,3\% dos pacientes avaliados no presente estudo apresentaram lesões oculares.

A maior parte das mães de recém-nascidos com SCZ e alterações oftalmológicas referiu sintomas no primeiro trimestre da gestação, com uma prevalência variando entre $55,7-85,7 \% .^{10,17,18} \mathrm{Na}$ presente amostra, 84,4\% referiram sintomas de infecção durante a gestação, sendo 62,9\% no primeiro trimestre. Tal fato, pode sugerir que a infecção neste período se associa com maior prevalência de malformações, semelhante a história natural de outras infecções verticais como toxoplasmose, rubéola e citomegalovírus. ${ }^{18}$ Outros fatores como a viremia e resposta imunológica da mãe e do feto também pode ter um papel importante na gravidade dessas anormalidades apresentadas. ${ }^{18}$

A idade gestacional média de realização do exame oftalmológico foi de 4.9 meses (1,2 e 9 meses), compatível com a literatura publicada com o mesmo público alvo $(5,7$ meses $\pm 0,9$ meses $).{ }^{19}$

Como outros flavivírus neurotrópicos, o ZIKV pode atingir os olhos a partir do cérebro, o primeiro local invadido, por transporte retrógrado via trato do nervo óptico, ou pode ter disseminação hematogênica por meio da barreira hemato-retiniana, como demonstrado em modelos animais. ${ }^{20}$ Nos olhos, o vírus parece ter maior predileção para alterações na câmara posterior, acometendo não apenas o nervo óptico, como disco óptico, retina e vasos retinianos. ${ }^{21} \mathrm{Em}$ nossa amostra, a atrofia macular e o mottling foram mais prevalentes, corroborando achados similares por outros autores. ${ }^{14-23}$ Não

\section{REFERÊNCIAS}

1. Plourde AR, Bloch EM. A literature review of Zika vírus. Emerg Infect Dis. 2016;22(7):1185-92.

2. Goeijenbier M, Slobbe L, Van Der Eijk A, Melo MM. Zika virus and the current outbreak: an overview. Neth J Med. 2016;(3):104-9.

3. Venancio FA, Bernal ME, Ramos MC, Chaves NR, Hendges MV, foi encontrada nenhuma alteração na câmara anterior, como catarata, uveíte, ou acometimento do cristalino, que apesar de possível, é pouco descrito nesses pacientes, resultado também semelhante ao descrito em outros trabalhos. ${ }^{23-24}$

A única evidência estatística observada neste estudo foi que os pacientes com alteração ocular apresentaram maior idade gestacional comparado aos sem alterações. Este estudo tem como limitações ter sido realizado como uma amostra por conveniência, com um pequeno número de pacientes, analisados em apenas um hospital, não sendo possível afirmar quais porcentagens dos achados podem ser generalizáveis para a infecção presumida pelo ZIKV na população em geral.

A avaliação oftalmológica foi realizada através de demanda espontânea de pacientes triados pelo grupo de pediatria que os acompanhava previamente, em serviço especializado no HUOL, sendo feita por preceptores e residentes, não sendo possível padronizar os achados, tampouco comparar avaliações intra ou inter observadores. Além disso, o diagnóstico oftalmológico foi avaliado segundo registro em prontuário específico dos pacientes, não sendo possível dispor de registro de imagem das fundoscopias realizadas.

\section{CONCLUSÕES}

Estudos sobre portadores de SCZ com alterações oculares ainda são escassos devido a doença ser relativamente nova em território nacional, além da dificuldade de triar e manter o seguimento com registro adequado desses pacientes em ambulatórios específicos.

Apesar do ápice das infecções ter ocorrido há dois anos, é importante traçar o perfil epidemiológico das alterações decorrentes da SCZ, para melhor entendimento dos mecanismos inerentes, de modo a estabelecer prioridades junto ao ensino e a pesquisa além do acesso à saúde da população, especialmente no Rio Grande do Norte.

As lesões oftalmológicas surgem como fator potencializador na dificuldade de estímulo e de aprendizagem na vida dessas crianças. Portanto, descrever as principais alterações neste grupo pode guiar profissionais no diagnóstico das lesões mais prevalentes. A realização do exame oftalmológico mostra-se essencial para o diagnóstico precoce, objetivando a correção de lesões potencialmente curáveis, no contexto da assistência completa. Além disso, é necessário o seguimento ambulatorial com equipe multidisciplinar e multiprofissional para garantir uma melhor qualidade de vida.
Souza MM, et al. Congenital zika syndrome in a Brazil-ParaguaiBolívia border region: clinical features of cases diagnosed between 2015 and 2018. PLoS One. 2019;14(10):1-17.

4. Brasil. Ministério da Saúde. Secretaria de Vigilância em Saúde. Monitoramento dos casos de dengue, febre de chikungunya e febre pelo vírus Zika até a Semana Epidemiológica 50, 2017. 
Boletim Epidemiológico. 2017 [acesso em: 4 nov 2019];48(45):113. Disponível em: http://portalarquivos2.saude.gov.br/images/ pdf/2018/janeiro/10/2017-046-Publicacao.pdf

5. Musso D, Ko AI, Baud D. Zika vírus infection - after the pandemic. N Engl J Med. 2019;381(15):1444-57.

6. Roma JH, Alves RC, Silva VS, Ferreira MJ, Araújo C, Pavoni JH. Descriptive study of suspected congenital zika syndrome cases during the 2015-2016 epidemic in Brazil. Rev Soc Bras Med Trop. 2019;52:1-8.

7. Carod-Artal FJ. Neurological complications of Zika virus infection. Expert Rev Anti Infect Ther. 2018;16(5):399-410.

8. Pool KL, Adachi K, Karnezis S, Salamon N, Romero T, NielsenSaines K, et al. Association between neonatal neuroimaging and clinical outcomes in Zika-exposed infants from Rio de Janeiro, Brazil. JAMA Netw Open. 2019;2(7):1-14.

9. Ventura CV, Ventura LO. Ophtalmologic manifestations associated with Zika vírus infection. Pediatrics. 2019;141(2):161-6.

10. Freitas BP, Dias JR, Prazeres J, Sacramento GA, Ko AI, Maia M, et al. Ocular findings in infants with microcephaly associated with presumed Zika vírus congenital infection in Salvador, Brazil. JAMA Ophtalmol. 2016;134(5):529-35.

11. Brasil. Ministério da Saúde. Secretaria de Vigilância em Saúde. Secretaria de Atenção à Saúde. Orientações integradas de vigilância e atenção à saúde no âmbito da Emergência de Saúde Pública de Importância Nacional: procedimentos para o monitoramento das alterações no crescimento e desenvolvimento a partir da gestação até a primeira infância, relacionadas à infecção pelo vírus Zika e outras etiologias infeciosas dentro da capacidade operacional do SUS. Brasília: Ministério da Saúde; 2017. 158 p.

12. World Health Organization. Implementation of resolutions and decisions. Infant and young child nutrition: the WHO multicentre growth reference study. 1999 [acesso em: 10 nov 2019]. Disponível em: https://apps.who.int/iris/bitstream/handle/10665/79006/eeid1. pdf? sequence $=1 \&$ is Allowed $=y$

13. World Health Organization. WHO child growth standards: methods and development - lenght/height-for-age, weight-for-age, weightfor-lenght, weight-for-height and body mass index-for-age. Geneva: WHO; 2006 [acesso em: 10 nov 2019]. 312 p. Disponível em: https:// www.who.int/childgrowth/standards/Technical_report.pdf?ua=1
14. Sahiner F, Sig AK, Savasçi U, Tekin K, Akay F. Zika vírusassociated ocular and neurologic disorders: the emergence of new evidence. Pediatr Infect Dis J. 2017;36(12):341-6.

15. Ventura CV, Maia M, Travassos SB, Martins TT, Patriota F, Nunes $\mathrm{ME}$, et al. Risk factors associated with the ophthalmoscopic findings identified in infants with presumed zika virus congenital infection. JAMA Ophthalmol. 2016;134(8):912-8.

16. Nithiyanantham SF, Badawi A. Maternal infection with Zika vírus and prevalence of congenital disorders in infants: systematica review and meta-analysis. Can J Public Health. 2019;110(5):63848 .

17. Venturi G, Fortuna C, Alves RM, Paschoal AG, Silva PJ Júnior, Remoli ME, et al. Epidemiological and clinical suspicion of congenital Zika vírus infection: serological findings in mothers and children from Brazil. J Med Virol. 2019;91(9):1577-83.

18. Ventura CV, Maia M, Ventura BV, van der Linden V, Araújo $\mathrm{EB}$, Ramos RC, et al. Ophtalmological findings in infants with microcephaly and presumable intra-uterus Zika vírus infection. Arq Bras Oftalmol. 2016;79(1):1-3.

19. Ventura LO, Ventura CV, Lawrence L, van der Linden V, van der Linden A, Gois AL, et al. Visual impairment in children with congenital Zika syndrome. J AAPOS. 2017;21(4):295-9.

20. Miner JJ, Sene A, Richner JM, Smith AM, Santeford A, Ban N, et al. Zika vírus infection in mice causes panuveitis with shedding of vírus tears. Cell Rep. 2016;16(12):3208-18.

21. Fernandez MP, Saad EP, Martinez MO, Corchuello S, Reyes MM, Herrera MJ, et al. Ocular histopathologic features of congenital Zika Syndrome. JAMA Ophthalmol. 2017;135(11):1163-9.

22. Benzekri R, Belfort R Júnior, Ventura CV, Freitas BP, Maia M, Leite $\mathrm{M}$, et al. [Ocular manifestations of Zika virus: what we do and do not know]. J Fr Ophtalmol. 2017;40(2):138-45. Article in French.

23. Guevara JG, Agarwal-Sinha S. Ocular abnormalities in congenital Zika syndrome: a case report, and review of the literature. J Med Case Rep. 2018;12(1):161-6.

24. Dias JR, Ventura CV, Freitas BP, Prazeres J, Ventura LO, BravoFilho V, et al. Ocular abnormalities in congenital Zika syndrome: are the ophthalmoscopic findings "the top of the iceberg"? Prog Retin Eye Res. 2018;66:85-106.

\section{Como citar:}

Andrade PY, Arrais NM, Maia CR, Rebouças CJ, Garrido RJ. Manifestações oculares em crianças com síndrome congênita do vírus Zika. Rev Med UFC. 2020 out-dez;60(4):25-31. 